\title{
A new perspective on causality, locality, and duality in entangled quantum nano systems
}

Sarma Gullapalli

Sarma N. Gullapalli, "A new perspective on causality, locality, and duality in entangled quantum nano systems," Proc. SPIE 10926, Quantum Sensing and Nano Electronics and Photonics XVI, 109260N (1 February 2019); doi: $10.1117 / 12.2506801$

SPIE. Event: SPIE OPTO, 2019, San Francisco, California, United States 


\title{
A new perspective on causality, locality and duality in entangled quantum nano systems

\author{
Sarma N. Gullapalli ${ }^{*_{a}}$
} \\ ${ }^{a}$ Retired Consultant, USA
}

\begin{abstract}
Interference with or without entanglement has been recognized as a key resource for quantum computing and quantum communications systems, as for example discussed by Nielsen and Chuang ${ }^{1}$ and numerous other works. Multiple paths between sources and detectors require an understanding of the underpinning wave-particle duality issue in the interference effects. Recently a new axiom (particle and its wave function $\psi(r, t)$ cannot be coincident or co-located at space-time point $\left(\mathrm{r}_{\mathrm{k}}, \mathrm{t}_{\mathrm{k}}\right)$ unless $\psi(\mathrm{r}, \mathrm{t})=\delta\left(\mathrm{r}-\mathrm{r}_{\mathrm{k}}, \mathrm{t}-\mathrm{t}_{\mathrm{k}}\right)$ the Dirac delta function) has been suggested $^{2}$ and justified, which explains duality without Niels Bohr's complementarity principle, thus eliminating the role of the observer, avoiding complicated "which way" (welcher-weg) considerations and observer subjectivity. This greatly simplifies analysis and design of multi-path quantum systems and restores objectivity. The same paper also suggested in the context of entanglement new concepts of (a) "total causality" that includes entanglement as a cause to locally and causally explain "action at a distance", and (b) "partial causality" that excludes entanglement as a cause and thereby introduces the perception of strange phenomena of non-locality, retro-causality and quantum erasure, which are nevertheless very important. This paper reviews and then applies the axiom to bring much needed clarity to certain confusing and much debated aspects of developments in non-interaction measurements, counterfactual communications and quantum computers. These potential clarifications and simplifications of analysis and design of multi path systems may help developers of future quantum communication and quantum computer systems.
\end{abstract}

Keywords: Quantum mechanics, Quantum communications, Quantum computers, Non-interaction measurements, Counterfactual communications, New Axiom for Duality, Interference, Complementarity

\section{INTRODUCTION}

Interference, viewed as superposition of states, is a key resource of quantum communications and quantum computer systems. This naturally involves the question of wave-particle duality, regarding which the wellestablished complementarity principle (due to Niels Bohr) states that one can observe either the wave nature or the particle nature of the quantum object (such as a photon or electron or ion) but not both simultaneously at any given space-time point. That is, the observation of particle nature destroys interference, and the observation of wave nature precludes particle behavior. Here the states of the measuring instrument (used for observation) are naturally included in the overall quantum system comprising of the observed quantum object and the measuring instrument such as polarizing beam splitter/detector or interferometer/detector system, similar to including the loading or termination effects of measuring apparatus in a classical system. But Bohr's complementarity principle is not just about such inclusion of states of measuring device in the overall quantum system, it is mainly about the act of observation influencing the measured object's wave or particle behavior. Albert Einstein disagreed with Bohr ${ }^{3}$, resulting in intense debates between the two, but Bohr's view has been confirmed by numerous ingenious experiments with and without entanglement ${ }^{4,5,6}$ to cite just a few. Discussions in this context of what exactly constitutes observation has resulted in epistemological suggestions to include also the consciousness of the person conducting the experiment, as for example suggested by Von Neumann, opening the door to even weirder metaphysical speculations, seriously degrading the objective clarity of science with unwarranted subjectivity, resulting in considerable confusion. J.S. Bell, a widely acclaimed quantum physicist in recent times, excludes

sngullapalli@hotmail.com Phone 703-862-2961 Address 6098 Creekview Ct, Harrisburg, NC 28075

Quantum Sensing and Nano Electronics and Photonics XVI, edited by Manijeh Razeghi,

Jay S. Lewis, Eric Tournié, Giti A. Khodaparast, Proc. of SPIE Vol. 10926, 109260N

(c) 2019 SPIE · CCC code: 0277-786X/19/\$18 - doi: 10.1117/12.2506801 
consciousness from observation on page 170 of his book?: "But I see no evidence that it is so in the success of contemporary quantum theory", an opinion which we must take seriously.

In a recent paper ${ }^{2}$ it has been shown, with justifying logic, that duality can be explained without Bohr's complementarity principle or "which way" observation, and the various experiments that confirm Bohr's complementarity principle, with or without entanglement, can be explained in terms of coherence and alignment alone, without the need for any mystical intelligence on the part of the inanimate particle to know about the nature of observation measurement, and it is shown that

Coherence and alignment $\equiv$ interference $\equiv$ no "which way" observation;

No coherence or alignment $\equiv$ no interference $\equiv$ "which way" observation

That is, "which way" observation (complementarity principle) is redundant, unnecessarily opens the door to unwarranted metaphysical speculations such as including the subjective consciousness of the observer, best avoided for clarity of objective science. This also greatly helps proper understanding of hotly debated subjects of noninteraction measurements and counter-factual communication. Next we state the Axiom with rationale, to set the stage for its applications to quantum communication and quantum computer systems. For details see paper ${ }^{2}$.

\subsection{Justified new Axiom² for quantum mechanics:}

Given that $|\psi(\underline{\mathbf{r}}, \mathrm{t})|^{2}$ defines the probability that the particle, an indivisible real quantum object, is at spacetime point $(\underline{\mathbf{r}}, \mathrm{t})$, where $\psi(\underline{\mathbf{r}}, \mathrm{t})$ is the particle's divisible complex probability amplitude wave (packet) function, we may define the co-location (coincidence) of particle and its wave function as unique co-location (coincidence) of centroid (or any such specified geometrical point) of particle with that of wave (packet).

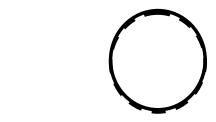

At point of coincidence probability $|\psi|^{2}<1$

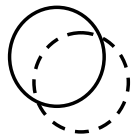

There are other points where $|w|^{2}<1$

(a) $|\psi|^{2}$ (dotted line) is not a delta function; $\psi$ and particle have same distribution

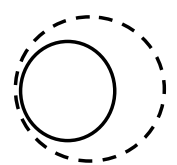

Particle at some point where $|\psi|^{2}<1$

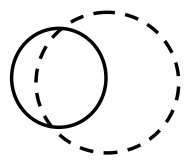

Particle at some other point where $|\psi|^{2}<1$

(b) $|\psi|^{2}$ (dotted line)

is not a delta function; $\psi$ and particle have different distributions

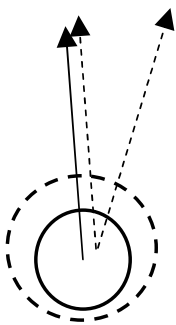

(c) $|\psi|^{2}$ (dotted line) is not a delta function; Divisible wave function $\psi$ can be on multiple paths, Indivisible particle can be on only one path

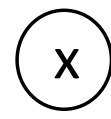

(d) $|\psi(\underline{\mathbf{r}}, \mathrm{t})|^{2}$

$=\delta\left(\underline{\mathbf{r}}-\underline{\mathbf{r}}_{0}, \mathrm{t}-\mathrm{t}_{0}\right)$ Centroid of $|\psi|^{2}$ and particle coincide at only one point

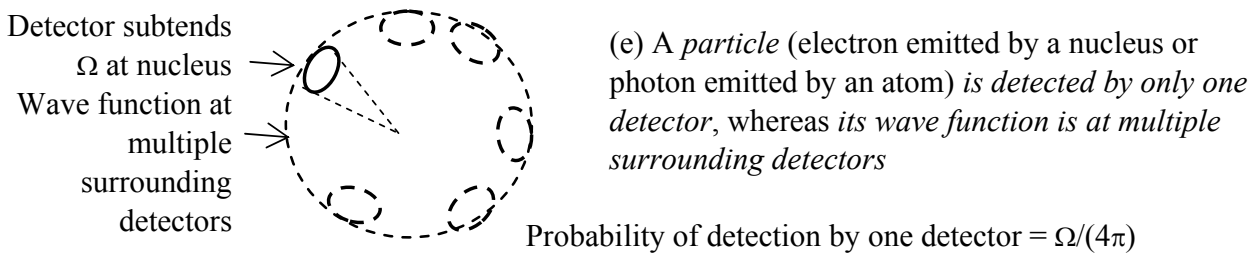

Figure 1. Coincidence / Colocation is possible at $\left(r_{0}, t_{0}\right)$ if only if $|\psi|^{2}$ is delta function $\delta\left(\underline{r}-r_{0} t-t_{0}\right)$

AXIOM: Wave function $\psi$ can be co-located (coincident) with its particle only at space-time points $\left(\underline{\mathbf{r}}_{0}, \mathrm{t}_{0}\right)$ where probability $\left|\psi\left(\underline{\mathbf{r}}_{0}, \mathrm{t}_{0}\right)\right|^{2}=\delta\left(\underline{\mathbf{r}}-\underline{\mathbf{r}}_{0}, \mathrm{t}-\mathrm{t}_{0}\right)$, the unit Dirac delta function at $\left(\underline{\mathbf{r}}_{0}, \mathrm{t}_{0}\right)$. At any space-time point where $|\psi|^{2}<$ 1 wave function cannot be uniquely co-located (coincident) with its particle. In $\mathrm{N}$-entangled system co-location 
(coincidence) applies to each entangled particle with its part of the joint wave function, possible only at respective space-time points where magnitude squared is respective delta function, the overall integral being unity.

Rationale for this Axiom is illustrated in Figure 1, (a) when $|\psi|^{2}$ is not a delta function but $\psi$ and particle have same distribution (b) when $|\psi|^{2}$ is not a delta function but particle and $\psi$ have different distributions, (c) when $|\psi|^{2}$ is not a delta function and there are multiple paths as in an interferometer, and (d) when $|\psi|^{2}$ is a delta function. Cases (a), (b) and (c) contradict $\psi$ being probability amplitude, according to which particle (its centroid) can probably be at different space-time points ( $\underline{\mathbf{r}}, \mathrm{t})$ where $1>|\psi(\mathrm{r}, \mathrm{t})|^{2}>0$. It is only when $|\psi|^{2}$ is a delta function as in (d) that the wave function and particle can be co-located (coincident). This fundamental distinction namely that particle and its wave function are two totally different aspects (physical particle and its non-physical wave function) and cannot be different interpretations of the same physical entity, is also clearly illustrated in practical situation (e) a particle (electron emitted by a nucleus or a photon emitted by an atom) is detected by only one detector out of many surrounding detectors whereas its wave function is at many surrounding detectors. Physical particle is local and its non-physical (complex probability amplitude) wave function is not local. Quantum Field Theory (QFT) also cannot satisfactorily explain local nature of particle and non-local nature of its wave function. Our Axiom separates the two; there is no need for particle to mystically morph into wave function or vice versa.

It may be noted that in arriving at this Axiom there are no new assumptions made, only new reasoning. It may also be noted that $|\psi(\underline{\mathbf{r}}, \mathrm{t})|^{2}=\delta\left(\underline{\mathbf{r}}-\underline{\mathbf{r}}_{0}, \mathrm{t}-\mathrm{t}_{0}\right)$ is satisfied at the position and instant $\left(\underline{\mathbf{r}}_{0}, \mathrm{t}_{0}\right)$ of creation of the particle-wave function such as when a photon is emitted by an atomic electron, and at the position and instant of annihilation of particle-wave function such as at total absorption of photon at detection. It may also be noted that the conventional view of wave function as a probability amplitude interpretation of some physical aspect of the particle, an assumption made in the context of duality, contradicts probability as shown in Figure 1, and so must be discarded.

An important consequence of this Axiom is that wave function always remains a wave propagating along all possible paths defining probabilities for various paths even when path configuration changes dynamically, while particle always remains particle following one particular path from among all probable paths. We show how this greatly simplifies and clarifies analysis of multi-path quantum communication and quantum computer systems.

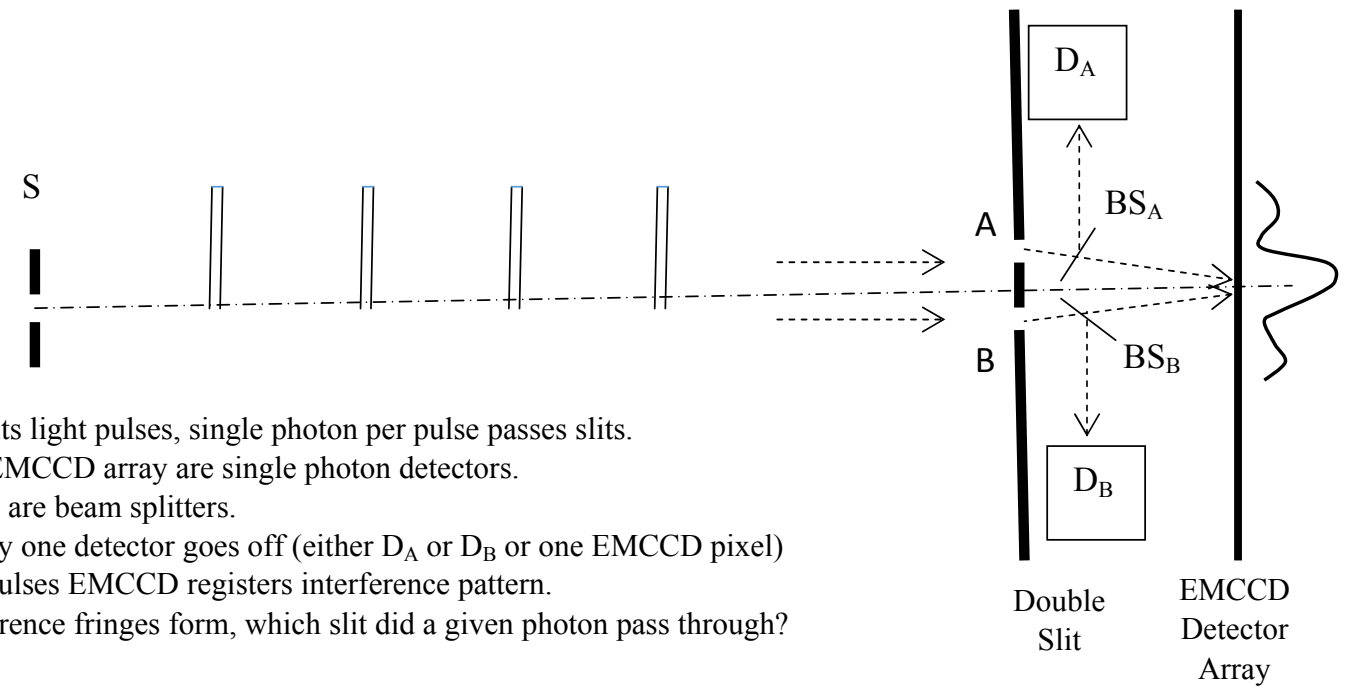

Figure 2. Young's double slit experiment with single photons; "which way?" question

Use of our Axiom is best illustrated by the fundamentally important generic single photon Young's double slit experiment shown in Figure 2. Applying our Axiom, divisible wave function remains wave passing through both 
slits, defining probabilities for paths to $\mathrm{D}_{\mathrm{A}}, \mathrm{D}_{\mathrm{B}}$ and to $\mathrm{EMCCD}$ where wave components from both paths interfere defining probability at each pixel; indivisible particle (photon) remains particle, goes through only one slit to only one detector. There is no mysterious wave-particle transformation caused by "observation". Interference is fully explained by coherence and alignment between the two paths (including alignment of polarization in case polarized photon is used and beam slitters are polarized).

It has been widely recognized that the wave function, a complex probability amplitude, is a purely mathematical construct, not a physical entity. For example, there is no bell shaped physical object corresponding to a Gaussian probability function characterizing some population. But the non-physical wave function evolves in space-time according to Schrodinger's wave equation for particle with non-zero rest mass such as an electron, and according to Schrodinger-Klein-Gordon wave equation for particle with zero rest mass such as a photon. In either case, physical parameters of the particle and medium are parameters in Schrodinger's equation, resulting in nonphysical wave function evolving according to physical parameters of particle and the medium. Why this is so (why Schrodinger's wave equation works) is the real mystery of quantum mechanics, worthy of rephrasing Albert Einstein's famous question "Is quantum mechanics complete?" to "Why (not how) does Schrodinger's wave equation (or equivalent Heisenberg's formalism) work?"

In fact, but for the interaction of non-physical wave function with physical objects (such as mirrors, beam splitters and detectors) and consequent superposition of probability amplitudes, there can be no interferometer! This fact and above Axiom help us understand with significantly better clarity and insight the current hot topics of noninteraction measurement, non-factual communication and duality computer, as we shall show.

\subsection{Quantum entanglement, causality and locality ${ }^{2}$}

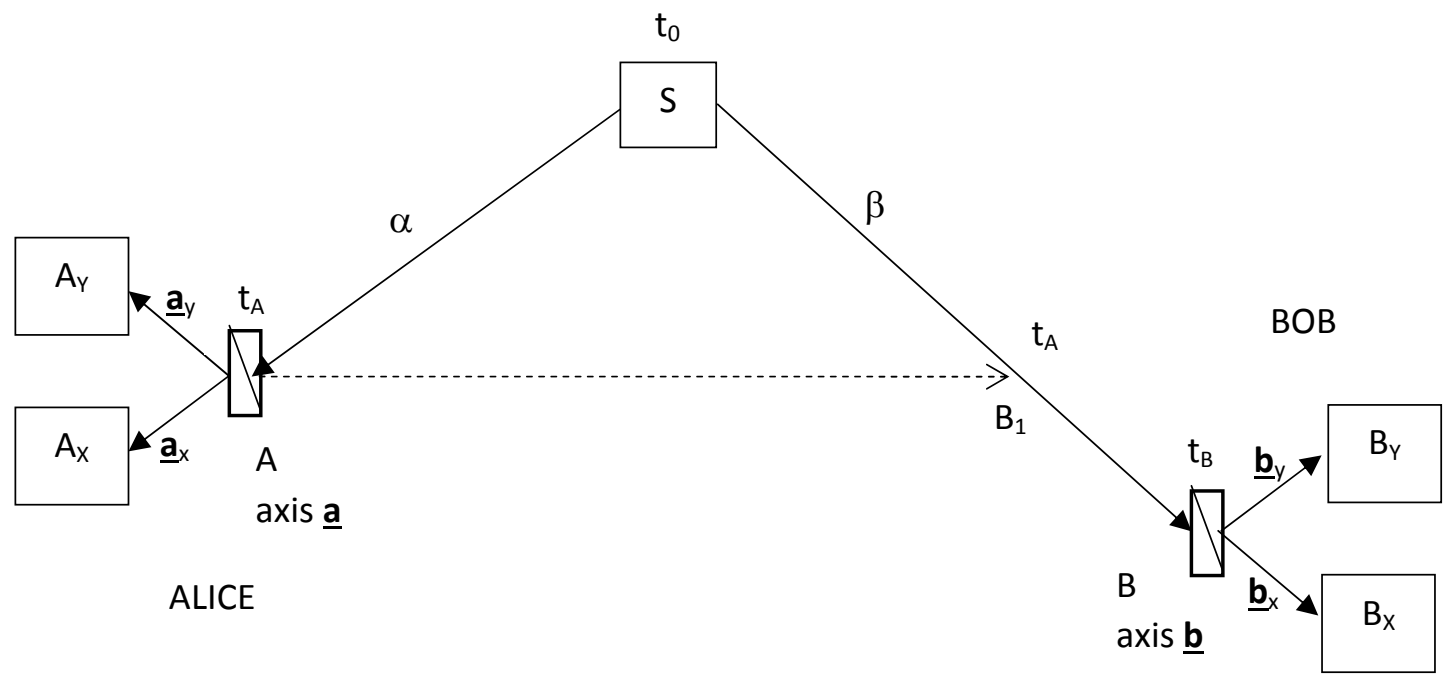

Figure 3. Entangled pair of particles $(\alpha, \beta)$ evolve causally from source $S$ which is the cause of entanglement

For its promise for secure communications, entanglement has become a key resource for quantum communication systems. It is also basic to quantum teleportation that evokes one's imagination and speculation. It originated in the famous E.P.R. paper ${ }^{8}$ with immediate response by N. Bohr' ${ }^{9}$. The term entanglement was coined by Schrodinger in his immediate response ${ }^{10}$ namely that entanglement is inherent in the very concept of probability amplitude nature of wave function. Entanglement has significantly altered our understanding of causality and locality. In addition to non-local action at a distance, it has introduced new experimentally demonstrated phenomena of retro-causality and quantum-erasure ${ }^{6,7}$, which have generated considerable discussions as to what exactly is causality ${ }^{11,12}$. 
This subject is also discussed in precedent paper ${ }^{2}$, where, for better clarity, new terms total causality and partial causality have been proposed. Total causality includes the source of entanglement also in the cause along with actions of Alice and Bob, whereas partial causality excludes the source of entanglement in the cause and considers only actions of Alice and Bob. The basic entanglement issues are illustrated in Figure 2, a widely used generic schematic. At time $t_{0}$ source $S$ creates and sends an entangled pair of particles $(\alpha, \beta)$ along spatially separated paths towards measurement stations, at $A$ at time $t_{A}$ for particle $\alpha$, and at $B$ at time $t_{B}>t_{A}$ for particle $\beta$. Due to entanglement, there is an entanglement constraint between the measured states of $\alpha$ and $\beta$ (such as polarization states of the two entangled photons). It is usually assumed that the state of $\beta$ is defined by the state of $\alpha$ instantly at time $\mathrm{t}_{\mathrm{A}}$ while $\beta$ is still at some point $\mathrm{B}_{1}$ enroute to $\mathrm{B}$. If measurement of $\alpha$ at $\mathrm{t}_{\mathrm{A}}$ is taken to be the cause and the instantaneous definition of state of $\beta$ is taken to be its effect, one concludes that there is non-local action at a distance, as the distance from $\mathrm{A}$ to $\mathrm{B}_{1}$ is not zero, and theory of relativity limits locality to distance covered by speed of light in free space. But ...

1. Without source $\mathrm{S}$ there is no entanglement constraint between states of particles $\alpha$ and $\beta$, and so $\mathrm{S}$ must be included in the total picture of causality (total causality) which is then causal and local with respect to $\mathrm{S}$.

2. If we exclude $\mathrm{S}$ from the picture, then in this partial picture there is non-local action at a distance (partial causality)

3. One cannot assume that measurement at $\mathrm{A}$ of particle $\alpha$ at time $\mathrm{t}_{\mathrm{A}}$ has an instantaneous effect on the state of particle $\beta$, because there is no measurement at point $\mathrm{B}_{1}$, and the effect of entanglement is known only when particle $\beta$ is measured at $B$ at time $t_{B}>t_{A}$. Therefore, space-time separated measurements at $\mathrm{A}$ and $\mathrm{B}$ must be considered together as one composite measurement that satisfies the entanglement constraint. If we regard measurement at B as (partial) cause and measurement at A as its effect, then we have retro-causality. Measurement of the pair is done only once, and so there is really no "erasure" of prior measurement.

In any communication system, causality is fundamentally important: In non-relativistic time sequence, sender must always precede the receiver, as otherwise the very meaning of "sender" and "receiver" is lost - sender must be the cause of communication and receiver must be the effect. This has always been the case with nonrelativistic classical communications. But in quantum communication system using entanglement, referring to Figure 3, if $t_{B}<t_{A}$ then the measurement choice of B decides the information and so B becomes the sender and $A$ the receiver! Therefore, times $t_{\mathrm{A}}$ and $\mathrm{t}_{\mathrm{B}}$ with respect to time $t_{0}$ at entanglement source $S$ are critical to determining who is sender and who is receiver. Therefore, in this causality picture we cannot ignore the entanglement source $\mathrm{S}$, with respect to which both $\mathrm{A}$ and $\mathrm{B}$ are causal.

\section{INTERACTION-FREE MEASUREMENTS (IFM) OF QUANTUM OBJECTS}

Interaction-free measurements with potential to preserve the state of a quantum signal particle, if feasible, would be valuable in quantum communications. In 1981, R.H. Dicke ${ }^{13}$ proposed "interaction-free measurement" through a thought experiment shown in Figure 4, as perhaps a paradox (?). For details see his paper ${ }^{12}$.

An intense light pulse is sent through the wave function of a trapped ion and any photons scattered by the ion are monitored by detectors completely surrounding the wave function (detectors not shown in the figure). If no scattered photons are detected, it is assumed that the wave function in the path of light pulse can be zeroed out, thus claiming that the negative result of non-interaction results in a modification of the wave function. R.M. Angelo ${ }^{14}$ points out the weakness in Dicke's reasoning as due to not recognizing the wave function as a probability function. But Dicke does recognize wave function as a probability function but seems to consider it as a probability interpretation of a physical aspect of the particle (ion), just as most scientists had viewed duality all along.

Applying our Axiom: Because wave function of ion is not a Dirac delta function at any point in the path of the light pulse, we cannot expect the particle to be there definitively when probed by the light pulse. More light pulses will eventually interact with the ion, provided the ion-photon interaction cross section is not zero. 
The zeroing of the wave function in the probe path due to negative result of the light pulse probe, thereby expecting the same negative result for any number of more light pulses, is actually a contradiction, not a paradox: If an arbitrarily large number of intense light pulses were all to produce negative result, then the assumption of the shape of the original wave function itself is wrong.

This example illustrates how the persisting notion that wave function is a probability amplitude interpretation of something physical associated with the particle is misleading. The wave function is simply not a physical entity.
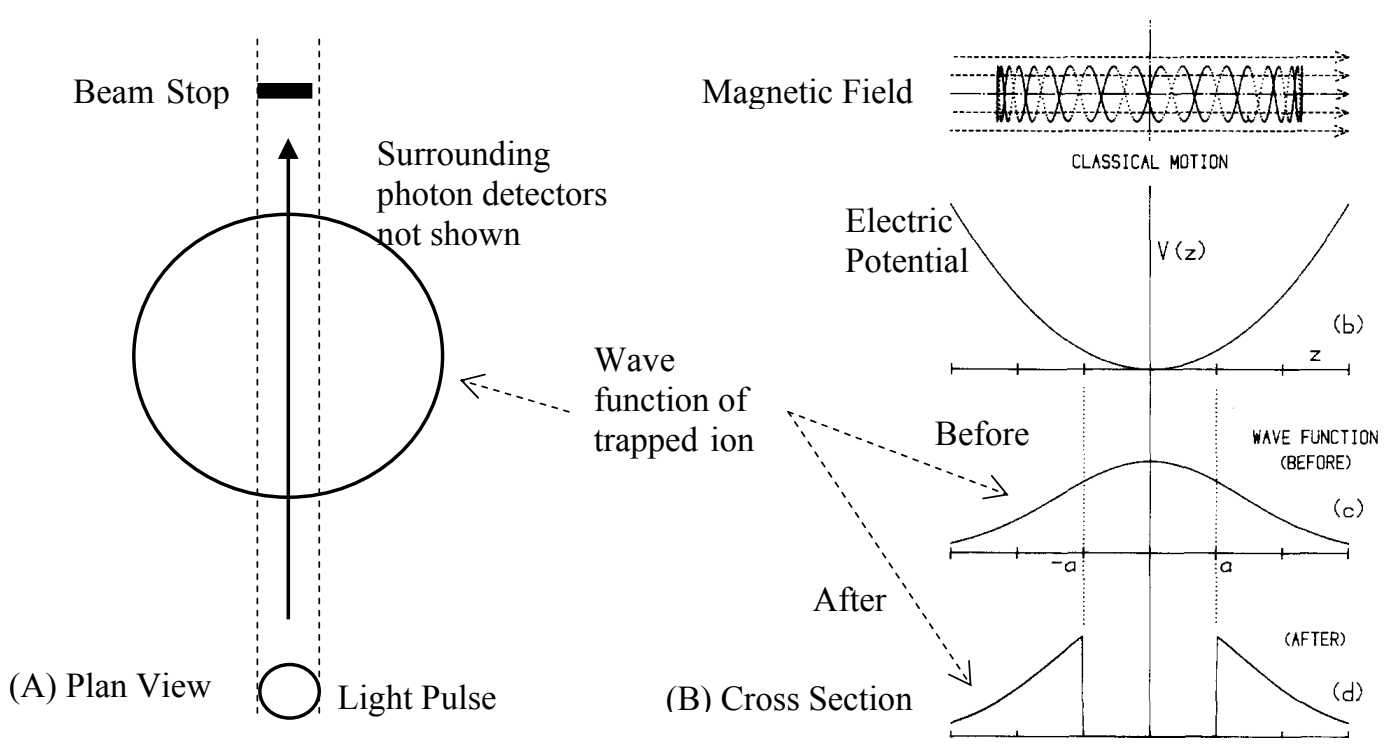

Figure 4. Thought experiment of R.H.Dicke ${ }^{13}$ for interaction-free quantum measurement: a paradox?

(Reuse of Figure 3 of R.H. Dicke's paper per Creative Commons License Attribution 4.0 terms of use at https://www.scitations.org; https://aapt.scitation/doi/10.1119/1.12592; )

Figure 5 shows another thought experiment for interaction-free quantum measurement, based on MachZehnder interferometer, proposed by A.C. Elitzur and L. Vaidman ${ }^{15}$. This attracted considerable debate and also attention for some forms of counterfactual communication which we shall discuss shortly. Vaidman has extensively reviewed these discussions ${ }^{16}$.

Referring to Figure $5, \mathrm{~S}$ is a source of single photons. $\mathrm{M}_{1}$ and $\mathrm{M}_{2}$ are ideal mirrors. $\mathrm{BS}_{1}$ and $\mathrm{BS}_{2}$ are $50 \%$ ideal beam splitters. $D_{1}$ and $D_{2}$ are ideal single photon detectors. Without the absorbing object $O$ in path2, the path lengths are such that constructive interference occurs at $D_{1}$ (count) and destructive interference at $D_{2}$ (no count). If now path2 is blocked by an absorbing object $\mathrm{O}$ (shown in dotted lines), then $\mathrm{D}_{1}$ or $\mathrm{D}_{2}$ will register count each with $25 \%$ probability $\left(50 \%\right.$ probability at $\mathrm{BS}_{1}$ for path 1 and $50 \%$ probability at $\mathrm{BS}_{2}$ for either $\mathrm{D}_{1}$ or $\left.\mathrm{D}_{2}\right)$. When detection of photon at $\mathrm{D}_{2}$ via path1 occurs, the presence of object $\mathrm{O}$ is thus sensed or "measured", without the photon interacting with object $O$, and thus it is claimed to be an interaction-free measurement.

Applying our Axiom: Wave function $\psi$ cannot be co-located with photon in either path1 or path2 because $|\psi|^{2}<1$ in either path due to $50 \% \mathrm{BS}_{1}$. Travelling both paths from $\mathrm{BS}_{1}$, wave function does interact with object $O$ in path 2. Thus it is incorrect to say that this is a truly interaction-free measurement. As discussed earlier, the unexplained mystery lies in Schrodinger's equation through which non-physical wave function depends on physical parameters. 
It can be said that quantum mechanics is more about the interactions of the particle's non-physical wave function with physical media (free space, mirrors, lenses, beam splitters, fibers, detectors, etc) covering the ensemble of all probable interactions of the physical particle with physical media (which is also behind all superposition and interference phenomena) than with a specific sample of interaction of the physical particle with physical media. The more general probabilistic ensemble interaction represented by wave function is more important, especially for interference and entanglement which are important resources for quantum communication and quantum computing.

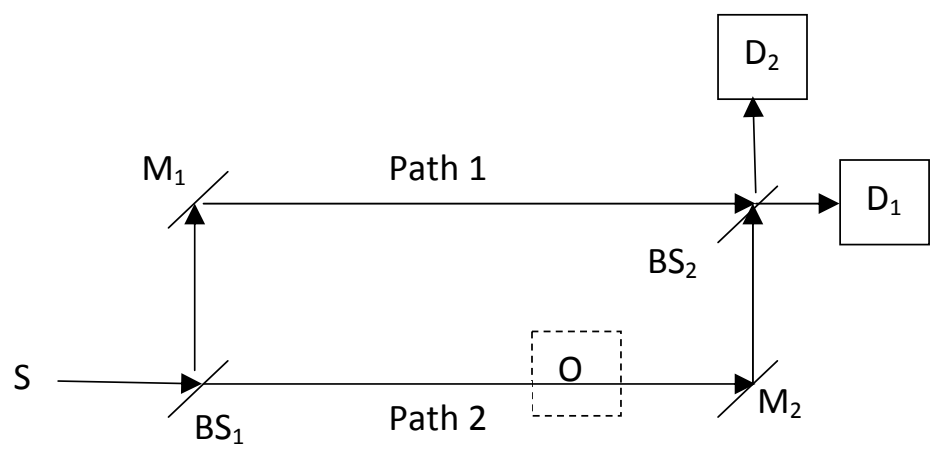

Figure 5. Elitzur and Vaidman (EV) proposal for interaction-free measurement ${ }^{15}$

It may be noted that the Elitzur - Vaidman proposal in Figure 5 is very similar to John Wheeler's delayed choice thought experiment (to test the role of observation in N. Bohr's complementarity principle to explain duality) described in figure 4 on page 183 of his book ${ }^{3}$, wherein it is the removal of $\mathrm{BS}_{2}$ that results in $\mathrm{D}_{1}$ registering count. Wheeler's delayed choice experiment has been extensively studied, and also discussed in the precedent paper ${ }^{2}$.

In an attempt at better terminology to describe the wave function, recently A. Shenoy, R. Srikanth ${ }^{17}$ have proposed to characterize the wave function as "real but non-physical". While this draws our attention to the dilemma, it is a bit confusing to say it is real but non-physical, because wave function is complex, not real.

As we shall see in section 3, recognition of this important fact: non-physical wave function does interact with physical media, throws a damper on the claims of counterfactual quantum communication also, which nevertheless are interesting and worth studying - but with some significant clarifying qualifications.

Figure 6 shows a Michelson interferometer version of the Mach-Zehnder interferometer based interactionfree measurement scheme of Elitzur - Vaidman (Figure 5), as this and its extensions will prepare us for discussions later of so-called counter factual communications with applications of our Axiom to gain a clearer understanding.

Source $\mathrm{S}$ sends a single photon into the interferometer. After going through beam splitters 2 and 1, path1 is to mirror $\mathrm{M}_{1}$ then back to beam splitter $\mathrm{BS}_{1}$ and then to detector $\mathrm{D}_{1}$. After beam splitters, path2 is to mirror $\mathrm{M}_{2}$ then back to $\mathrm{BS}_{1}$ then to $\mathrm{BS}_{2}$ and then to detector $\mathrm{D}_{2}$. Path lengths are such that constructive interference occurs at $\mathrm{D}_{1}$ (count) and destructive interference at $\mathrm{D}_{2}$ (no count). If now an absorbing object $\mathrm{O}$ is placed in path2, then interference is destroyed and either $\mathrm{D}_{1}$ or $\mathrm{D}_{2}$ registers count in the event photon does not go through path2 and get absorbed (if photon is absorbed by $\mathrm{O}$ neither $\mathrm{D}_{1}$ nor $\mathrm{D}_{2}$ can register count.). Thus, when $\mathrm{D}_{2}$ registers count object $\mathrm{O}$ is sensed ("measured") without photon interacting with the object, which may be claimed to be interaction-free measurement.

Applying our Axiom, as photon's wave function $\psi$ travels all possible paths and $|\psi|^{2}<1$ in all paths, photon cannot be co-located with wave function in any of the paths; wave function does travel path 2 and interact 
with object $\boldsymbol{O}$. Thus it is not exactly interaction-free, just as in the case of Elitzur-Vaidman Mach-Zehnder scheme in Figure 5.

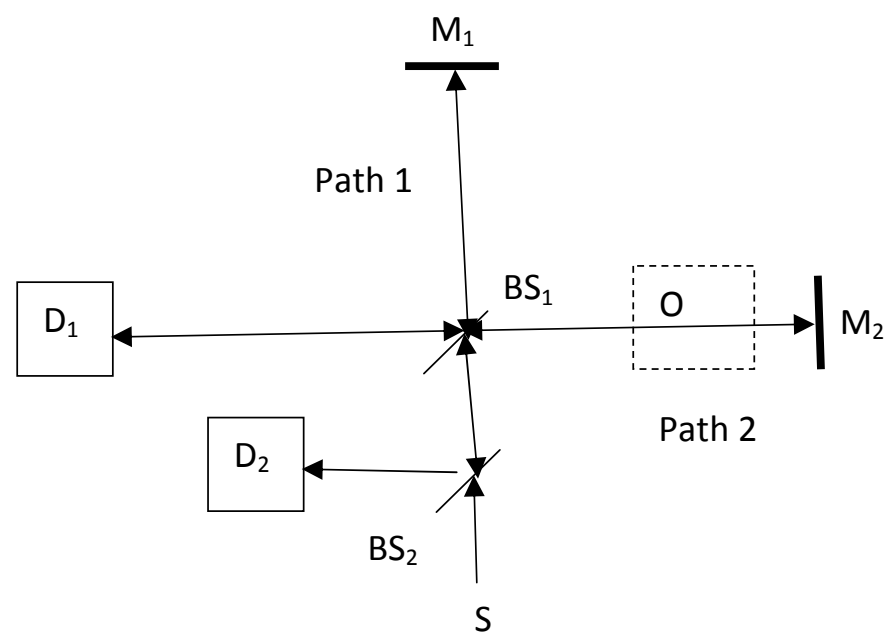

Figure 6. Michelson interferometer version of interaction-free measurement

\section{ENHANCING PROBABILITY OF IFM BY QUANTUM ZENO EFFECT}
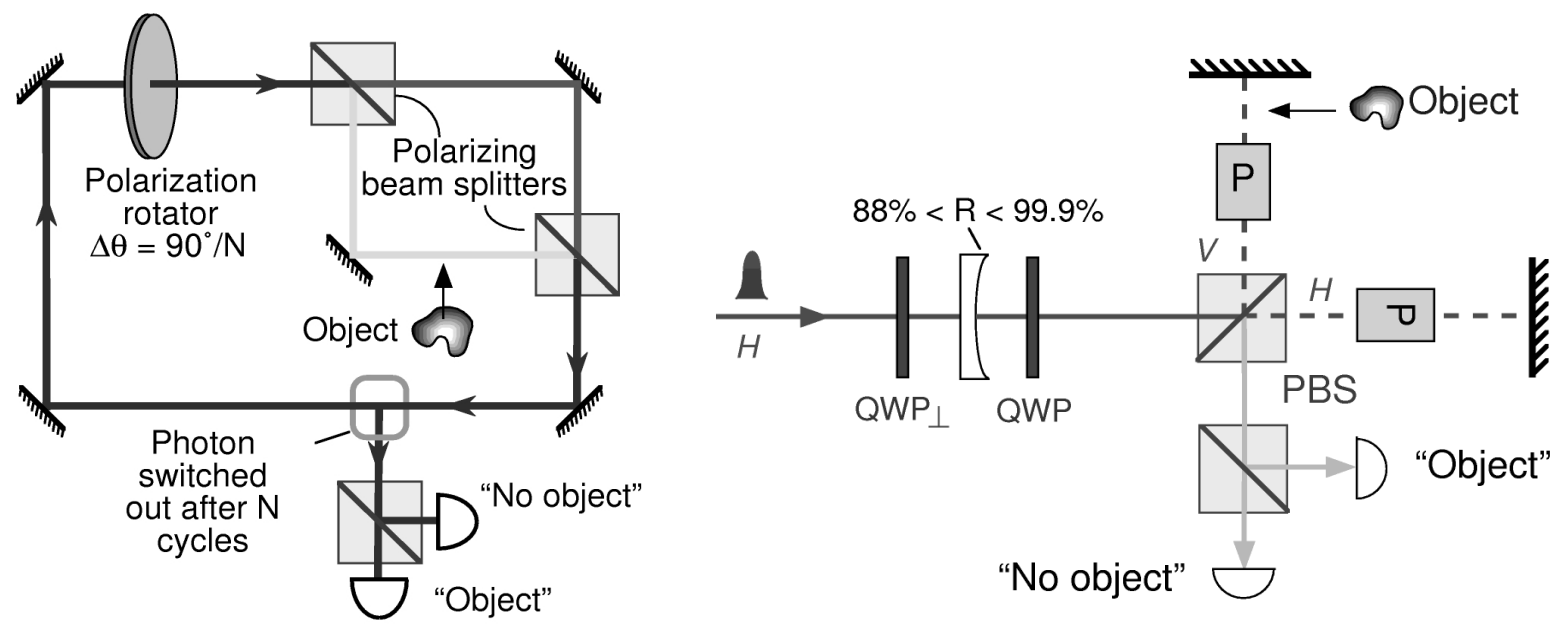

(a) Concept

In (a) injection of horizontally polarized particle at top left is not shown.

(b) Experiment

Polarizing beam splitter transmits $\mathrm{H}$ and reflects $\mathrm{V}$.

Figure 7. P.G. Kwiat, A. Zeilinger et $\mathrm{al}^{18}$ enhancement of IFM probability using Quantum Zeno Effect

(Figures 1 and 2 of their paper $^{18}$ reused under APS License RNP/18/NOV/009928)

In the Elitzur-Vaidman (EV) thought experiment for interaction free measurement (IFM) shown in Figure 5 , the probability of IFM is $25 \%$ of all trials, and $50 \%$ of trials in which detector $\mathrm{D}_{2}$ records count. In 1999, P.G. Kwiat, A.G. White, J.R. Mitchell, O. Nairz, G. Weihs, H. Weinfurter and A. Zeilinger ${ }^{18}$ reported a way to significantly boost the probability of IFM by using an optical version of Quantum Zeno Effect (concept discussed in 1977 by B. Misra and E.C.G. Sudarshan ${ }^{19}$ ), and verified it experimentally. 
Their concept and experimental setup are shown in Figure 7 (a) and (b) respectively, with permission to reuse by APS. For details see their paper ${ }^{18}$, in which they prefer to call the measurement as quantum interrogation instead of interaction free measurement, the basic idea being the same, namely sensing (interrogation or measurement) an absorbing object without the physical particle interacting with the object. Here we shall limit our discussion to aspects that are made clearer by application of our Axiom. Note that their concept (a) is based on Mach-Zehnder interferometer for convenience of explanation, while their experiment (b) is based on Michelson interferometer version for practical convenience. They are essentially equivalent. To understand the concept, a horizontally polarized photon is injected into the system at top left (injection not shown in figure (a)) and its plane of polarization is rotated by a small angle $\Delta \theta=\pi /(2 \cdot \mathrm{N})$, where $\mathrm{N}$ is a large number. $\mathrm{H}$ component probability amplitude $\cos (\Delta \theta)$ is transmitted by PBS while V component probability amplitude $\sin (\Delta \theta)$ is reflected. Note that by our Axiom it is the wave function $\psi$ (which defines probability amplitudes for various paths) that is split, not the particle (photon) which follows only one path, and since $|\psi|^{2}<1$ for either path, particle cannot be co-located (coincident) with wave function $\psi$ in either path.

Now, (i) when the absorbing object does not obstruct the path, the $\mathrm{V}$ and $\mathrm{H}$ components of probability amplitude coherently combine (assuming path lengths are equal and stable) to form the resultant $\psi$ at the output of second PBS with the same polarization angle as at the input of the first $P B S$, which is $\Delta \theta$ for the first time around the loop. For the second time around the loop the polarization angle is $2 \Delta \theta$ and so on, for the $\mathrm{m}$-th cycle, $\mathrm{m}<\mathrm{N}$, it is $\mathrm{m} \Delta \theta$. After $\mathrm{N}$ cycles, when polarization angle is $\pi / 2$, the path to detectors is switched on for the wave function (and the photon), and because polarization is now $\mathrm{V}$, the "No object" detector must record a count with probability 1.

On the other hand, (ii) when the absorbing object blocks the path, in the first cycle the small V component probability amplitude $\sin (\Delta \theta)$ of wave function is absorbed by the object, and so it does not reach the second PBS, and the $\mathrm{H}$ component probability amplitude $\cos (\Delta \theta)$ of wave function is transmitted as such through the second PBS. In the second cycle, this $H$ component gets rotated by $\Delta \theta$ and so the angle of polarization remains at $\Delta \theta$. Indeed, angle of polarization remains at $\Delta \theta$ through all cycles - this state of polarization as defined by angle of polarization remaining unaltered is termed Zeno effect, named after the paradox discussed by ancient Greek philosopher Zeno ${ }^{20}$. Also, after $\mathrm{N}$ cycles when the path to detectors is switched on, due to the H polarization of wave function, it is transmitted to "Object" detector which must record a count with probability $\cos ^{\mathrm{N}}(\Delta \theta)$, which tends to 1 in the limit as $N$ tends to $\infty$.

Thus we see that this system, without particle interacting with the object, detects absence of object with probability 1 , and presence of object with probability close to 1 , tending to 1 as $\mathrm{N} \rightarrow \infty$, which is truly remarkable. Moreover, it accomplishes this with arbitrarily small amplitude of wave function reaching the object as $\mathrm{N}$ is made arbitrarily large. Here we have a paradoxical situation that in the theoretical limit the object has a significant effect on the system with not only the particle, but also the wave function, not interacting with it! Researchers have noted this paradoxical situation in theory, though in practice it is difficult to make $\mathrm{N}$ arbitrarily large.

We shall now show that it is impossible, even in theory, to make the number of cycles $N$ arbitrarily large. Let $\mathrm{L}_{0}$ be the optical path length of the loop, the distance traveled by wave function and photon in one cycle. Then time taken for $\mathrm{N}$ cycles is $\mathrm{T}_{\mathrm{N}}=\mathrm{N} \cdot \mathrm{L}_{0} / \mathrm{c}$, where $\mathrm{c}$ is velocity of light. Therefore, for given time $\mathrm{T}_{\mathrm{N}}$ per measurement,

$$
\mathrm{N} \leq \mathrm{T}_{\mathrm{N}} \cdot \mathrm{c} / \mathrm{L}_{0}
$$

Because $\mathrm{L}_{0}$ cannot be made arbitrarily small, $\mathrm{N}$ cannot be made arbitrarily large in any finite time $\mathrm{T}_{\mathrm{N}}$. As a consequence of $\mathrm{N}$ being finite, wave function always interacts with the object when it blocks the path. There is no paradox. We note that this resolution is not possible if one assumes that the particle (photon) and its wave function are one and the same, as in conventional view of duality per complementarity principle. This resolution and clarity is possible only due to our Axiom which does away with complementarity principle and maintains that wave function always remains a wave (defining probabilities of evolution along various paths and superposition and interference) and particle (photon) always remains a particle, following only one particular path. We shall see next that this clarification significantly limits claims of "counterfactual" quantum communication. 


\section{4. “COUNTERFACTUAL" QUANTUM COMMUNICATION}

In classical communication, information (bit) is encoded in a physical entity such as macro electromagnetic wave carrier sent from sender to receiver. In quantum communication, the information (qbit) is encoded in the state of a physical object such as a particle, usually a photon, which is transmitted from sender (Alice) to receiver (Bob). In recently developed "counterfactual" quantum communication using ingenious schemes, communication is claimed to be achieved without any physical particle passing from sender (Alice) to receiver (Bob) through the communication channel. Because physical particle is susceptible to be intercepted by eavesdropper (Eve), it is claimed that "counterfactual" quantum communication holds promise for increased security. We show these tall claims fall short.
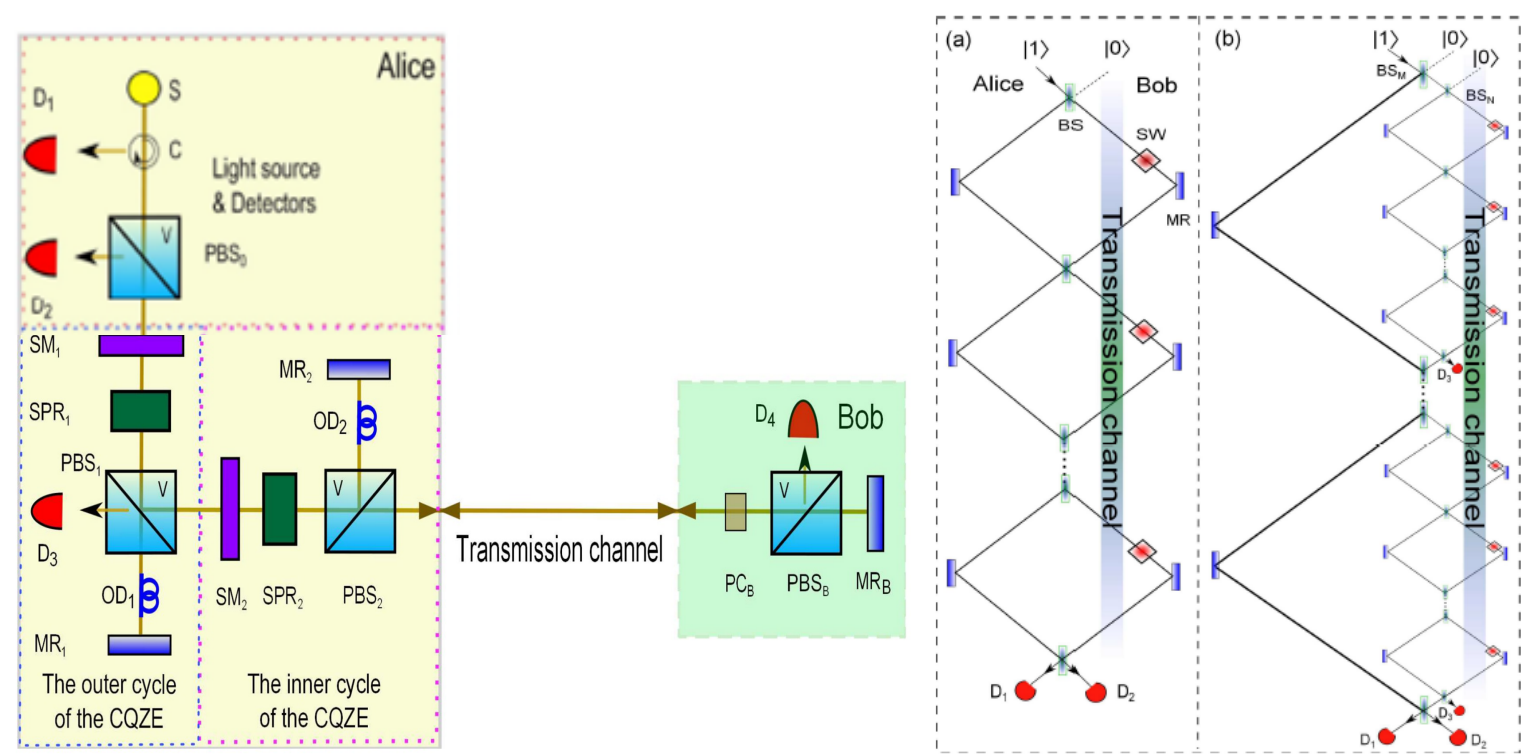

Figure 8. SLAZ (Salih, Li, Amri, Zubairy) scheme ${ }^{21}$ for counterfactual communication

(Reuse of Figures 1 and 2 of SLAZ paper ${ }^{21}$ under APS license RNP/18/NOV/009925)

In 2013, H. Salih, Z.H. Li, M. Al-Amri and M.S. Zubairy ${ }^{21}$ (SLAZ) proposed a thought experiment for counterfactual communication using interferometers, conceptually similar the quantum interrogation (interaction free measurement) scheme using quantum Zeno effect proposed in 1999 by Kwiat and Zeilinger ${ }^{18}$ which we have already discussed, but with the function of "switch" at the end of $\mathrm{N}$ cycles (at which time photon passes to final detectors) replaced with an additional $\mathrm{M}$ cycles for each of the main $\mathrm{N}$ cycles (claimed to avoid photon traversing communication channel at the end of $\mathrm{N}$ cycles). Their scheme, shown in Figure 8, has come under strong criticism $^{22}$, ${ }^{23}$. In 2017 Cheng-Zhi Peng, Jian-Wei Pan et $\mathrm{al}^{24}$ reported implementing a limited version of SLAZ experimentally and claim to have successfully verified their results against predictions. Also, SLAZ has been patented ${ }^{25}$ in USA.

On left is the Michelson interferometer version proposed for practical implementation. On right is the Mach-Zehnder version with opened up loops for conceptual understanding, (a) without inner cycles wherein photon is said to traverse communication channel at the end, (b) with inner cycles wherein photon is claimed not to ever traverse the communication channel as $\mathrm{N} \rightarrow \infty$. Details of controversial claims made is outside the scope of this paper. We shall limit ourselves to how the clarity of this concept (or any such concept) is improved by our axiom.

For example, SLAZ paper ${ }^{21}$ states (italics by author to highlight the contradiction) "with the help of pockel cell $\mathrm{PC}_{\mathrm{B}}$ Bob can either switch the polarization of incoming $H$ photon to a $\mathrm{V}$ photon or keep the polarization state unchanged. The $\mathrm{PBS}_{\mathrm{B}}$ reflects $\mathrm{V}$ photons to a detector $\mathrm{D}_{4}$ (effectively blocking the communication channel) and 
allows $\mathrm{H}$ photons to be reflected back by the mirror $\mathrm{MR}_{\mathrm{B}}$. Bob can send a stream of logic 0 's and 1's by either keeping the polarization state $\mathrm{H}$ unchanged (logic 0 ) or switching it to polarization state $\mathrm{V}$ (logic 1). Bob's choice of logic 0 and 1 leads to a click at detectors $\mathrm{D}_{1}$ and $\mathrm{D}_{2}$ respectively with almost unit probability and with almost no photon in the transmission channel, thus leading to counterfactual communication" - there is the incoming photon, but no photon in the communication channel! This contradiction is due to the conventional view of duality that particle changes to wave and vice versa, and can be cleared by applying our Axiom, by which wave function always remains wave traveling all possible paths defining various probabilities while particle (photon) always remains particle traveling a single particular path to $D_{1}$ or $D_{2}$ without traversing communication channel, assuming validity of probability computations - debate of which is outside scope of this paper. Thus it is the wave function and not particle that is incoming to Bob during the many $(\mathrm{N} \cdot \mathrm{M})$ cycles defining the probabilities for the photon which takes a single particular path to detector $\mathrm{D}_{1}$ or $\mathrm{D}_{2}$.

Note that Bob has to keep his selection of 0 or 1 for the entire duration $T_{N}$ of $N$ outer cycles for each bit ( $\mathrm{N} \cdot \mathrm{M}$ inner cycles), $\mathrm{T}_{\mathrm{N}}=\mathrm{N} \cdot \mathrm{L}_{0} / \mathrm{c}$ where $\mathrm{L}_{0}$ is the optical path length per one outer cycle and $\mathrm{c}$ is velocity of light. This means the bit rate is limited to $1 / \mathrm{T}_{\mathrm{N}}$ which is $\mathrm{c} /\left(\mathrm{N} \cdot \mathrm{L}_{0}\right)$ which tends to zero as $\mathrm{N} \rightarrow \infty$. This is a serious limitation of SLAZ scheme or any other scheme using interferometric quantum Zeno effect for quantum communication. Also, timing by Alice must be synchronized with Bob's, and path differences must be stabilized to a small fraction of wavelength.

Following conventional view of duality (that particle changes to wave and vice versa) the paper ${ }^{24}$ on successful experimental realization of SLAZ with $\mathrm{M}=4, \mathrm{~N}=2$, states that "photon reaching Bob is discarded when absorber is selected by Bob", thus implying that photon does traverse the communication channel, contradicting their statements "when single photons are used the counterfactual property is preserved in the case of logic 0 for a finite $M$ and $N$ " and "(even when $N$ is small) the counterfactual property is preserved for the case of logic 1 in all practical scenarios". This contradiction is resolved by our axiom, by which it is the wave function, not particle that is absorbed. As shown in Figure 3 of their paper ${ }^{24}$ the measured probability (for 0 or 1 signal) drops to about $82 \%$ for $\mathrm{M}=4, \mathrm{~N}=2$, which is an impressive improvement from $50 \%$ without quantum Zeno effect. However, it took more than 5 hours to transmit 10 kilobits, slowed not only by the minimum time per bit $\left(\mathrm{T}_{\mathrm{N}}=\mathrm{N} \cdot \mathrm{L}_{0} / \mathrm{c}\right)$ but also by the $52 \mathrm{~dB}$ channel loss which required Alice to repeat each bit several times.

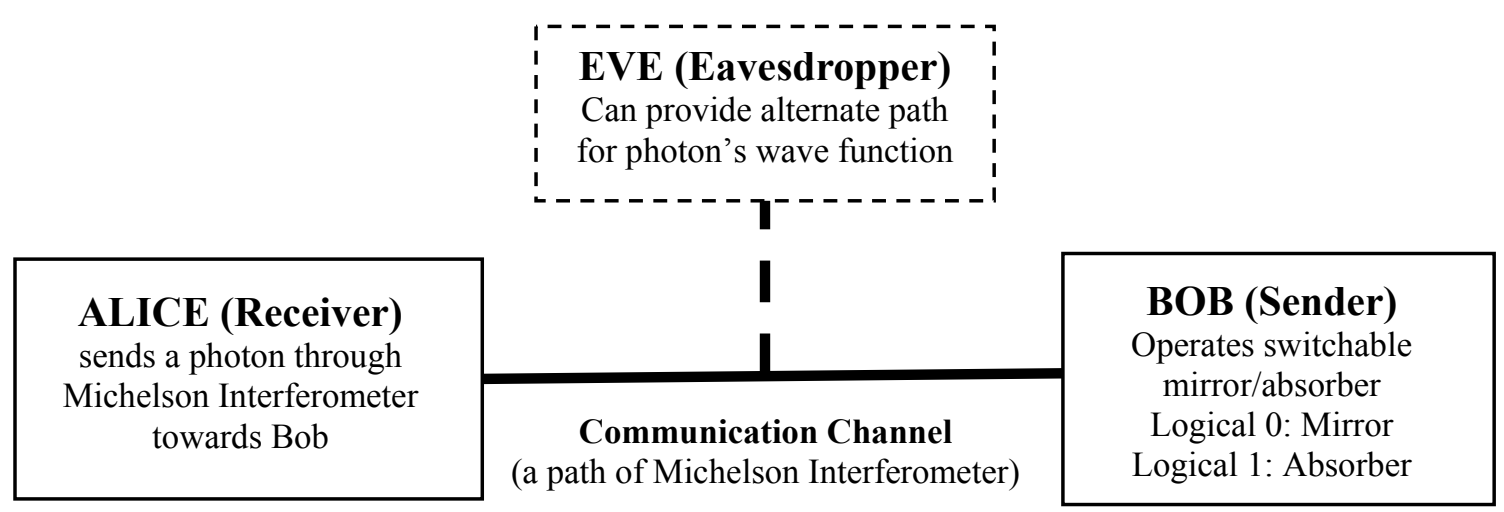

Figure 9. Wave function does interact with objects: a susceptibility of counterfactual communication

Thus, SLAZ scheme seems to have serious limitations. Also, stabilization of optical path differences to small fraction of wavelength necessitates auxiliary active control loops, a challenge even between adjacent optical benches, a formidable task over long distance communication links.

As shown generically in Figure 9, due to the fact that the non-physical wave function does interact with physical objects, a whole class of such counterfactual schemes including the SLAZ are really not interaction-free or in general free from Eve's eavesdropping interference. Given that Eve can access the channel (otherwise 
eavesdropping is impossible), such as by a coupler or beam splitter, Eve can provide an alternate path for the nonphysical wave function (which explores all possible paths) even when physical photon is not traversing the channel, and thereby change the characteristics of the interferometer, possibly duplicate Alice. This susceptibility, combined with the formidable challenge of maintaining tight interferometric tolerances on path differences over long distances, would seem to seriously limit practical use. However, a review paper on quantum communication ${ }^{26}$ reports counterfactual communication (with interferometric tolerances) over a few $\mathrm{km}$.

\section{QUANTUM COMMUNICATION USING ENTANGLEMENT}

Experimental investigations of entanglement require sufficient physical separation of Bob's terminal from Alice's (Figure 3) to avoid any possibility of classical communication. An example ${ }^{6}$ with more than 140 kilometers separation was discussed in precedent paper $^{2}$ in the context of applying our Axiom to explain results without "which way" complementarity. Recently a joint China - Austria team has demonstrated quantum communication using entanglement via a satellite link ${ }^{27-30}$, clearly demonstrating feasibility over very long distances. For our discussion, which is limited treatment of duality that may arise in such systems, Figure 10 shows a very basic generic quantum communication system using entanglement. The point to be made here is that any use of interferometer is local to terminal and so there is no challenge of maintaining optical path lengths to interferometric tolerances over communication channel, in sharp contrast to SLAZ and other counterfactual communication systems.

Eve's interception will be a disturbance sensed by Bob in which case he voids data. Thus Eve can disrupt communication but not eavesdrop. If interferometer is used to process $\mathrm{H}$ and $\mathrm{V}$ polarizations locally in the terminal, then duality "which way" complementarity issue inevitably arises.

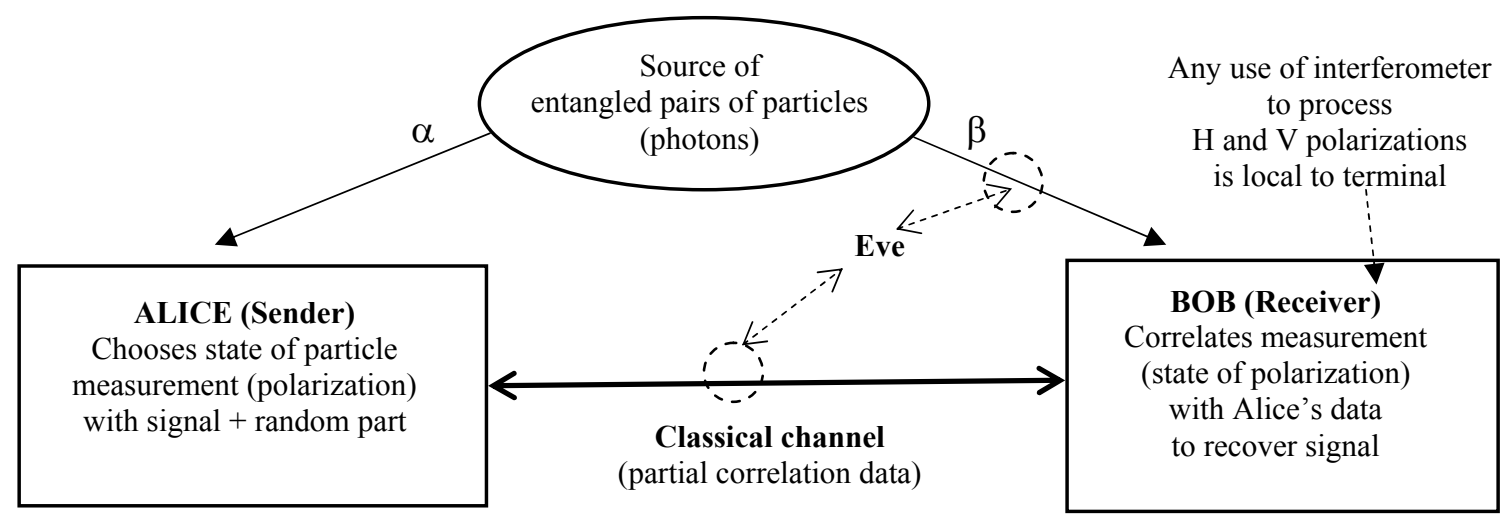

Figure 10. Quantum communication using entanglement

In all such cases application of our Axiom helps avoid "which way" observation complementarity issues, as wave function always remains wave defining probabilities for alternate paths while particle remains particle following a particular path out of the many probable path. It is hoped that this will clarify and simplify analysis and design of future terminal systems.

\section{DUALITY COMPUTER}

"Duality Computer" ${ }^{31}$ shown conceptually in Figure 11 is claimed to be more powerful than regular quantum computers because it utilizes parallel processing of "sub-waves" of the wave function. Stan Gudder ${ }^{32}$ provides a mathematical treatment of the duality computer concept. The ordinary quantum computer typically processes a single particle (such as single photon or electron) in such a way that the output measurements contain the result of computations. In the duality computer the input wave function $\psi_{\text {in }}$ of the particle is passed through a quantum wave divider, which is typically a set of slits (not beam splitters which may alter the state of input wave function) which outputs multiple attenuated copies $\psi_{1}, \psi_{2}, \psi_{3}$ etc of the input wave function without altering its 
state which are then processed by respective quantum processors the outputs of which are superposed in quantum wave combiner and measured. This parallelism of multiple processors processing the input wave function gives corresponding increase in computing power. It may be noted that there is no cloning involved in the quantum wave divider which is typically slits as in Young's double slit experiment. It may also be noted that this is not same as running several ordinary quantum computers in parallel, because here exactly the same input state is provided to each processors, which is not possible without cloning in separate ordinary quantum processors.

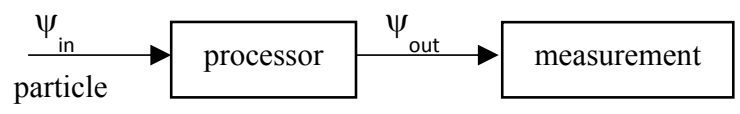

(a) Ordinary Quantum Computer

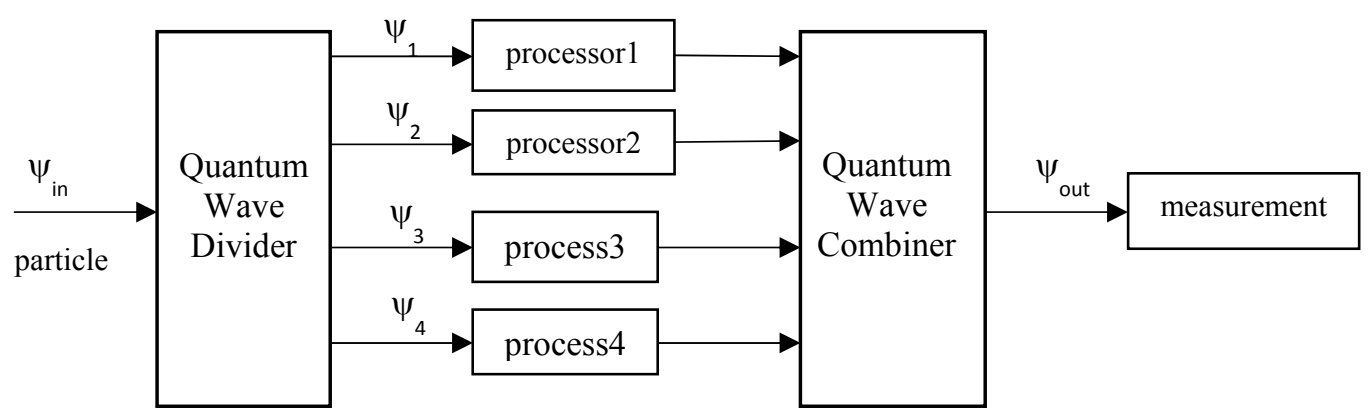

(b) Duality Computer

Figure 11. Duality Computer concept of Gui Lu Long ${ }^{32}$

However, in describing his duality computer concept for a three slit case, author Gui Lu Long says on page 5 of his paper $^{31}$ : "For instance a three slits wall will divide a wave into 3 parts, each with $1 / 3 \mid \psi>_{i}$ where the subscript $\mathrm{i}$ indicates the path number. However this information should not be available at the detector, otherwise the interference pattern will disappear" (italics by author). The last sentence is consequence of conventional complementarity view of duality, namely "which way" path information destroys interference, which is not only imprecise, bordering on mystical subjectivity than objective science, it makes systematic analysis and design of multi path systems complicated and confusing, if not impossible. It is in such situations, as complexity of future quantum computers inevitably increases with multitude of paths, that our Axiom finds useful application: Interference depends only on coherence and alignment (including alignment of polarizations) which can be systematically analyzed and designed for, there is no need to determine if "which way" path knowledge is implied in the measurements.

\section{NANO SCALE PHOTONIC QUANTUM COMPUTER CHIPS AND DUALITY}

Two major reported developments in quantum computer have been: (1) Electronic quantum computer using up/down spin of electron as the basic quantum state, but requiring strong magnetic fields and cryogenic temperatures, which point to highly centralized processing to afford the infrastructure (2) Photonic quantum computer using polarization and angular momentum of photon as the basic quantum states, but with poor linear inter-photon interaction. However, topological photonics seem to provide answer, quoting Dr Alberto Peruzzo of Australian Center of Excellence for Quantum Computation and Communication (CQC2T) ${ }^{33}$ about their topological photonic chip: "Topological photonics have the advantage of not requiring strong magnetic fields, and feature intrinsically high-coherence, room temperature operation and easy manipulation". 
Australian CQC2T is also reported ${ }^{34}$ to be developing electronic 10 -qubit quantum integrated circuit prototype in Silicon to be accomplished by 2022, but it requires superconducting magnets to provide the magnetic field needed to flip the spin states of electrons, and cryo temperature to reduce noise. In either case (electronic or photonic) CQC2T developments are examples of major advances in integrated quantum circuits (chips) at the nanoscale just as classical computer chips are at the nano-scale. Understanding what happens at the nano-scale (without the benefit of usual discrete components on an optical bench) becomes crucial. Research examples ${ }^{35,36}$ discuss multiquantum dot structures.

Our discussion is limited to duality issues that will inevitably arise as complexity of quantum computers increases using such chips. A basic component in photonic quantum computer is the beam splitter (CQC2T's topological photonic chip is claimed to replicate the functionality of beam splitters) which is typically used to separate the paths of incoming polarized photon on $\mathrm{H} / \mathrm{V}$ basis, and later to combine paths for interferometric superposition of states. There are also many single photon detectors in the system. According to conventional complementarity view of duality, the all-important interference (superposition) critically depends on whether or not some of the detectors constitute sensing "which way" the photon went, a task that can become highly complicated as the number of beam splitters, beam combiners and detectors increase, as one would expect in integrated quantum circuits (chips). Our axiom completely eliminates this complexity, as non-physical wave function remains wave throughout defining various probabilities of various paths and at various detectors, while physical photon follows only one particular path out of the many probable paths, with the particular probability. The equivalence established in precedent paper ${ }^{2}$

Coherence and alignment $\equiv$ interference $\equiv$ no "which way" observation;

No coherence or alignment $\equiv$ no interference $\equiv$ "which way" observation

allows us to avoid troublesome "interference $\equiv$ no "which way" observation" which can also involve observer's subjectivity, and work with objective "coherence and alignment $\equiv$ interference" enhancing clarity in analysis/design.

\section{CONCLUSION AND DISCUSSION}

The proposed justified Axiom is shown to provide much needed clarification in treating wave-particle duality in all quantum systems including quantum communication systems and quantum computers, by noting ${ }^{2}$ that particle and its wave function cannot be coincident or co-located except at space-time points where the wave function is a Dirac delta function such as at instant of creation and at instant of annihilation. This avoids "which way" (welcher-weg) complementarity criterion and resulting complexity in analysis and design of quantum communication systems and quantum computer systems. A result of the Axiom which rejects the notion that particle mysteriously turns to wave and vice versa, ensures that (a) wave function always remains wave exploring all possible paths defining probability amplitudes for various paths, and (b) particle always remains particle following one particular path out of all probable paths, greatly simplifies analysis and design of quantum systems, especially in future integrated photonic chips that hold promise for room temperature quantum computers and communication systems.

Future efforts will be to expand the applications of this Axiom to more areas, and to further explore quantum communication and quantum computer systems.

\section{REFERENCES}

[1] Michael A. Nielson and Isaac L. Chuang "Quantum Computation and Quantum Information”, Cambridge University Press (2010)

[2] Sarma N. Gullapalli "Explaining duality, the "only mystery" of quantum mechanics, without complementarity or "which way" (welcher-weg)" International Conference on Quantum Mechanics and Applications" July 20-21 Atlanta GA USA; archived at http://vixra.org/quant/1712.0558 
[3] J.A. Wheeler and W.H. Zurek [Quantum Theory and Measurement], Princeton University Press, 9-31 (1984)

[4] V.Jaques, E. Wu, F. Grosshans, F. Truessart, P. Grainger, A. Aspect, J.F.Roch "Experimental realization of Wheeler's delayed-choice Gedanken Experiment" OSA Conference on Coherence and Quantum Optics, Session XII CQ09, Rochester NY USA (2007); arXiv:quant-ph/061024v1 (28 Oct 2006)

[5] Yoon-Ho-Kim, R. Yu, S.P. Kulik, Y.H. Shih “A Delayed Choice Quantum Eraser” Physical Review Letters (2000); arXiv:quant-ph/9903047v1 (13 Mar 1999)

[6] Xiang Song Ma, Johannes Kofler, Angie Qarry, Nurey Tetik, Thomas Scheidl, Rupert Ursin, Sven Ramelow, Thomas Herbst, Lothar Ratschbacher, Alessandro Fedrizzi, Thomas Jennewein, Anton Zeilinger "Quantum erasure with causally disconnected choice" PNAS Vol 110, No.4, 1221-1226 (2013)

[7] J.S. Bell "Speakable and Unspeakable in Quantum Mechanics. Cambridge Univ. Press (1987)

[8] A. Einstein, B. Podolsky, N. Rosen "Can quantum-mechanical description of physical reality be considered complete?”, Physical Review 47 (May 15, 1935)

[9] N. Bohr "Can quantum-mechanical description of physical reality be considered complete?", Physical Review, Vol 48 (October 15, 1935)

[10] E. Schrodinger "Discussion of probability relations between separated systems" Mathematical Proceedings of Cambridge Philosophical Society, Vol 31, Issue 4 (October 1935)

[11] Ognyan Oreshkov, Fabio Costa, Caslav Bruckner "Quantum correlations with no causal order", Nature Communications, DOI: 10.1038/ncomms2076 (Oct 2012)

[12] Caslav Bruckner “Quantum Causality” Nature Physics Vol 10 (April 2014)

[13] R.H. Dicke "Interaction-free quantum measurements: A paradox?", American Journal of Physics, 49 (Oct 1981)

[14] Renoto M. Angelo "On the interpretive essence of the term "interaction-free measurement": The role of entanglement, arXiv:0802.3853v3 [quant-ph] 10 Dec 2008

[15] A.C. Elitzur and Lev Vaidman "Quantum mechanical interaction-free measurement" arXiv:hep-th/9305002v2 (5 May 1993)

[16] Lev Vaidman "The meaning of the interaction-free measurements", Foundations of Physics, Vol. 33, No. 3 (March 2003)

[17] A. Shenoy, R. Srikanth "The wave-function is real but nonphysical: A view from counterfactual quantum cryptography”, arXiv:1311.7127v2 [quant-ph] (7 July 2014)

[18] P.G. Kwiat, A.G. White, J.R. Mitchell, O. Nairz, G. Weihs, H. Weinfurter and A. Zeilinger "High-Efficiency Quantum Interrogation Measurements via the Quantum Zeno Effect" Physical Review Letters Vol.83, No.23 (6 December 1999)

[19] B. Misra and E.C.G. Sudarshan “The Zeno's paradox in quantum theory” J. Math. Phys. 18, 756 (1977)

[20] Jeff Speakes, Zeno paradoxes by Jeff Speakes.pdf, Univ. of Notre Dame course PHIL 13195

[21] H Salih, Z H Li, M Al-Amri, M S Zubairy "Protocol for Direct Counterfactual Quantum Communication" arXiv:1206.2042v5 [quant-ph] (24 April 2013); PhysRevLett.110.170502 (2013)

[22] L. Vaidman "Comment on "Protocol for Direct Counterfactual Quantum Communication"” arXiv:1304.6689v2 [quant-ph] (30 April 2013) 
[23] Sahli, Li, Al-Amri, Zubairy Reply, arXiv:1404.5392v2 [quant-ph] (28 April 2014)

[24] Yuan Cao, Yu-Huai Li, Zhu Cao, Juan Yin, Yu-Ao Chen, Hua-Lei Yin, Teng-Yun Chen, Xiongfeng Ma, Cheng-Zhi Peng, Jian-Wei Pan, "Direct counterfactual communication via quantum Zeno effect", PNAS, vol 114, no 19 (May 9, 2017)

[25] Patent US8891767B2 "Method and apparatus for direct counterfactual quantum communication" H Salih, Z H Li, M Al-Amri, M S Zubairy (December 21, 2012)

[26] A. Avella, G. Birda, D. Carpentras, A. Cavenna, I.P. Degiovanni, M. Genovese, M. Gramegna, P. Triana "Review of recent groundbreaking experiments on quantum communication with orthogonal states" arXiv:1206.1503v1 [quant-ph] 7 June 2012

[27] "Real-world intercontinental quantum communications enabled by the Micius satellite" 2018 January 19 phyics.org, https://phys.org/news/2018-01-real-world-intercontinental-quantum-enabled-micius.html

[28] Liao et al 2017, Nature 549, 43 satellite-to-ground decoy state QKD with $\mathrm{kHz}$ rate over a distance of $\sim 1200 \mathrm{~km}$

[29] Yin et al 2017, Science 356, 1140, Bell test

[30] Ren et al 2017, Nature 549, 70, ground-to-satellite quantum teleportation

[31] Gui Lu Wong “The General Quantum Interference Principle and the Duality Computer" Communications in Theoretical Physics vol 45 no 5 (2006) pp 825-844 / Arxiv: quant-ph/0512120

[32] Stan Gudder "Mathematical Theory of Duality Quantum Computers" Journal of Quantum Information Processing vol 6, issue 1, February 2007 pp 37-48

[33] "New photonic chip promises more robust quantum computers" 14 September 2018 https://phys.org/news/2018-09-photonic-chip-robust-quantum.html

[34] Brian Wang “10-qubit quantum integrated circuit prototype in silicon by 2022” NextBigFuture.com Nov 17, 2018

[35] M.C. Rogge, R.J. Huag "The three dimensional stability of triple quantum dot stability diagrams" New Journal of Physics, vol 11, no 11, 2009

[36] Sofia Olsson "Entanglement in Nano Systems" diploma work on bachelor level (supervisor Peter Samuelsson) Lund University faculty of science publication, 2014 